\title{
Joint Estimation for Incorporating MRI Anatomic Images into SPECT Reconstruction
}

\author{
Yong Zhang, Jeffrey A. Fessler, Neal H. Clinthorne, W. Leslie Rogers \\ Bioengineering Program, The University of Michigan
}

\begin{abstract}
To improve SPECT reconstruction using spatiallycorrelated magnetic resonance(MR) images as a source of side information, one must account for mismatch between MRI anatomical information and SPECT functional information. We investigate an approach which incorporates the anatomical information into SPECT reconstruction by using region labels representing the anatomical regions extracted from MRI. Each SPECT pixel corresponds to one region label. Both SPECT pixel mean intensities and region labels are jointly estimated by a penalized MaximumLikelihood criterion using an iterative Space-Alternating Generalized EM algorithm. The likelihood function incorporates both the SPECT noise distribution and the MRI side information measurement statistics. Since the region labels are estimated jointly from both segmented MRI and SPECT projection data, only those anatomical regions that match SPECT functional regions are represented by the estimated labels, and are used to constrain the SPECT reconstruction. The artifacts due to the mismatched MR anatomical region information are reduced using joint estimation. By comparing image quality and the Bias vs. Variance tradeoffs, we see that the joint estimation has the potential to improve the SPECT estimation result.
\end{abstract}

\section{INTRODUCTION}

The fluctuation of photon statistics, trade-offs between detection sensitivity and collimator resolution, and limited photon rates cause the inherently modest resolution in SPECT imaging. Since SPECT has the capability of disclosing the functional information in living organs, while other imaging modalities such as MRI give relatively higher resolution images, many efforts have been made to apply side-information, extracted from higher-resolution, structurally correlated MR images, to constrain the SPECT image prior in a Maximum a posteriori (MAP) objective to improve the image quality and quantitative accuracy. Two major approaches exist for incorporating MR anatomic information: line site models, which represent anatomic

This work was supported in part by NIH grant CA54362 and DOE grant DE-FG02-87ER60561 boundaries [1,2]; and labelling methods, which identify anatomic regions [4]. Both methods provide improved SPECT reconstruction if the anatomical structure is perfectly correlated with SPECT functional information. A direct method would use region labels to define the weights in a weighted Gibb's function as a penalty function in a penalized Maximum-likelihood objective [3], so intensity discrepancies are only penalized within the same region and not across region boundaries, as in case of spatiallyinvariant regularization. Such labels are treated like a prior, they influence the SPECT reconstruction. However, there may exist mismatches between MRI anatomical structure and SPECT functional structure since the spin density and $T_{1}, T_{2}$ distribution are not necessarily the same as SPECT tracer distribution. The "blind" use of MRI side information causes artifacts $[3,5]$. For example, if the segmented MRI is missing a corresponding functional region in SPECT, then the "blind" use of this anatomical information might suppress the reconstruction of the functional region. Some investigations have been done to accommodate this fact, such as Leahy's "functional line sites method" [1], Ouyang's "weighted lines sites" method [5], and Fessler's "blurred weights" method [3].

We investigate a joint estimation approach that incorporates the anatomic side information and its measurement statistics into SPECT image reconstruction to reduce the effect of imperfect side information. We choose region labels to represent the anatomical regions extracted from MRI because the region labels more easily favor region contiguity and need half less the parameters than line sites representations. In this approach, each SPECT pixel $\lambda_{k}, k=1, \ldots, p(p$ : number of pixels) corresponds to one region label $l_{k}, k=1, \ldots, p$. If two pixels have the same type of region labels, they tend to have the similar intensities, vice versa. However the region labels $l$ are not simply a direct "copy" from the segmented MRI. Instead, after being initialized with the segmented MRI, they are jointly estimated with SPECT pixel mean intensity parameters $\lambda$, using a penalized Maximum-Likelihood(PML) objective. We update a SPECT mean intensity pixel and its corresponding region label simultaneously, such that label and pixel pair updating are mutually constrained. The joint objective function incorporates both the SPECT noise distribution and the MRI side information measurement statistics, plus a joint penalty function, as:

$$
\boldsymbol{\Phi}_{\text {joint }}(\boldsymbol{\lambda}, \mathbf{l})=L(\boldsymbol{\lambda}, \mathbf{l} ; \mathbf{y}, \tilde{\mathbf{I}})-\Omega(\boldsymbol{\lambda}, \mathbf{l})
$$


where $\mathbf{y}$ is the SPECT projection data, $\tilde{\mathbf{l}}$ is the MR anatomic region measurement, $\boldsymbol{\lambda}$ and $\mathbf{l}$ are SPECT intensity and label parameters to be estimated, $L()$ and $\Omega()$ are joint likelihood and joint penalty terms. Since region labels are estimated jointly from both MRI and SPECT, only those parts of the MR anatomical regions that match the SPECT functional regions are represented by the estimated labels, and constrain the SPECT intensity reconstruction, while the mismatched region labels will be updated during the joint estimation process, to approach the SPECT functional region. Thus the artifacts due to mismatch are reduced.

Section 2 describes the method in detail. Section 3 gives simulation results comparing visual qualities of the reconstructed images and their bias vs. variance trade-offs. Some problems we encountered in applying the method and possible future study are described in Section 4 .

\section{METHODS}

\section{A. Objective Function}

We choose penalized Maximum-Likelihood as our objective, which can also be viewed as a Maximum a posteriori if regarding the penalty terms as an a priori pdf of possible image and label states from a Bayesian perspective. So the estimation of both SPECT intensity $\boldsymbol{\lambda}$ and region labels 1 will be

$$
(\hat{\lambda}, \hat{\mathbf{l}})=\arg \max _{\lambda, 1} \boldsymbol{\Phi}_{j \text { oint }}(\lambda, \mathbf{l}) .
$$

The objective function is shown as (1). Since SPECT and MRI are independent imaging processes, their joint loglikelihood functions are independent and can be separated, and we adopt a hierarchical penalty function. Thus the resulting penalized objective function is

$$
\boldsymbol{\Phi}_{\text {joint }}(\boldsymbol{\lambda}, \mathbf{l})=L(\boldsymbol{\lambda} ; \mathbf{y})+L(\mathbf{l} ; \tilde{\mathbf{l}})-\beta_{1} \Omega_{1}(\boldsymbol{\lambda} ; \mathbf{l})-\beta_{2} \Omega_{2}(\mathbf{l})
$$

where $\mathbf{y}, \tilde{\mathbf{l}}, \mathbf{l}, \boldsymbol{\lambda}$ are the same as before. $L(\boldsymbol{\lambda} ; \mathbf{y})$ is the $\log$ likelihood of SPECT, $L(\mathbf{l} ; \tilde{\mathbf{1}})$ is the log-likelihood of MR side information measurement statistics. $\Omega_{1}$ is the penalty term which relates the estimation of the labels 1 to the estimation of the intensity $\lambda$, and $\Omega_{2}$ is a penalty term which encourages contiguous regions. Applying the statistics of the side information measurement allows us to avoid "blind" use of MR side information. The SPECT measurement is well known to be Poisson process; the log-likelihood is given by [7]:

$$
L(\boldsymbol{\lambda}, \mathbf{y})=\log f(\mathbf{y} ; \boldsymbol{\lambda}) . \equiv \sum_{n}\left(-\bar{y}_{n}(\boldsymbol{\lambda})+y_{n} \log \bar{y}_{n}(\boldsymbol{\lambda})\right)
$$

where

$$
\bar{y}_{n}(\lambda)=\sum_{k} a_{n k} \lambda_{k}+r_{n}
$$

where " $\equiv$ " represents the equivalence, $r_{n}$ is the assumed known scatter. The log-likelihood of the side information
$L(\mathbf{1} ; \tilde{\mathbf{1}})$ depends on how the anatomical information is extracted from the MR images. For the reason of simplicity, we assume that the MRI has Gaussian noise and different regions have different intensities. If MRI is segmented using simple pixel-by-pixel thresholds, then the log-likelihood for $P\left(\tilde{l}_{k} \mid l_{k}\right)$ for $\tilde{l}_{k}$ given $l_{k}$ is:

$$
L(\mathbf{l} ; \tilde{\mathbf{l}})=\sum_{k=1}^{p} \log P\left(\tilde{l}_{k} \mid l_{k}\right)
$$

where $P\left(\tilde{l}_{k} \mid l_{k}\right)$ is the probability of assigning the label $\tilde{l}_{k}$ to the $k$ th pixel when $l_{k}$ is the true label. Each of these $P\left(\tilde{l}_{k} \mid l_{k}\right)$ can be empirically estimated from MRI noise statistics and structural information from both filteredbackprojection image of SPECT and segmented MRI. We think that certain amount of human interaction is necessary for determining whether there might be a mismatch between segmented MRI and SPECT tracer distribution. For example, in Fig. $1, P\left(\tilde{l}_{k}=A \mid l_{k}=B\right)$ can be determined by MRI noise statistics, however, whether there is a possible region $D$ in SPECT has to be checked using a FBP, and assign certain probability level. This term determines the extent to which we trust each region in the MRI side information.

The penalty terms restrict the possible states of SPECT image and region labels. To encourage smoothness within the same region but allow discontinuities between regions in the SPECT image, we choose $\Omega_{1}$ as:

$$
\Omega_{1}(\boldsymbol{\lambda}, \mathbf{l})=\frac{1}{2} \sum_{k=1}^{p} \sum_{j \in \mathcal{N}_{k}} \omega_{k j}(\mathbf{l}) \psi\left(\lambda_{k}-\lambda_{j}\right)
$$

with $\omega_{k j}(\mathbf{l})= \begin{cases}1 \text { (direct neighbor) } & \text { if } l_{k}=l_{j} \\ \sqrt{2} / 2 \text { (diagonal neighbor) } & \text { if } l_{k}=l_{j} \\ 0 & \text { if } l_{k} \neq l_{j}\end{cases}$

$\mathcal{N}_{k}$ is the neighborhood of pixel $k$, usually a 2nd-order eight-pixel neighborhood is used. The weights $\omega_{k j}$ link the pixel $k$ with its neighbors $j$, and $\psi\left(\lambda_{k}-\lambda_{j}\right)$ is usually a quadratic term. Here $\omega_{k j}$ is not fixed, it is a function of labels. When $\psi\left(\lambda_{k}-\lambda_{j}\right)$ is too big, the maximization pushes the current label $l_{k}$ to be different from its neighbors. This penalty term associates the SPECT intensity vector with the label vector, so their estimations are affected by each other, instead of letting the labels extracted from MR dominate the SPECT reconstruction. A possible form for $\Omega_{2}$ will be

$$
\Omega_{2}(\mathbf{l})=\sum_{k=1}^{p} \sum_{j \in \mathcal{N}_{k}} \omega_{k j}(\mathbf{l})
$$

where $\omega_{k j}$ is the same as above. This term encourages the neighboring pixels to have the same labels.

\section{B. Reconstruction Algorithm}

We apply an iterative Space-Alternating Generalized EM(SAGE) [6] algorithm for the penalized MaximumLikelihood objective because of its faster convergence and 
FBP

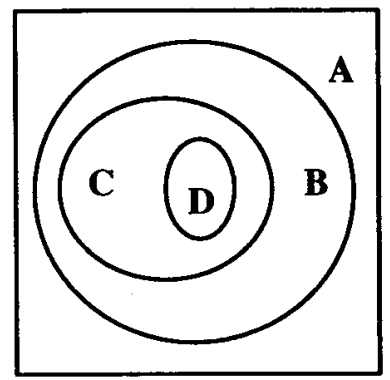

Figure 1: Compare FBP and MRI for $P\left(\tilde{l}_{k} \mid l_{k}\right)$.

its monotonicity. In SAGE, the penalized likelihood $\boldsymbol{\Phi}_{\text {joint }}(\boldsymbol{\lambda}, \mathbf{l})$ is maximized by maximizing $\phi^{k}\left(\lambda_{k}, l_{k} ; \lambda^{i}, \mathbf{l}^{i}\right)$, the sum of the conditional expectation $Q^{k}\left(\lambda_{k}, \lambda^{i}\right)$ of the log-likelihood and the penalty terms of the hidden-data space [6]. Each pixel-label pair is jointly updated by holding the remaining pixel-label pairs fixed:

$$
\begin{aligned}
& \left(\lambda_{k}^{i+1}, l_{k}^{i+1}\right)=\arg \max _{\left(\lambda_{k}, l_{k}\right)} \phi^{k}\left(\lambda_{k}, l_{k} ; \lambda^{i}, \mathbf{l}^{i}\right) \\
& \phi^{k}\left(\lambda_{k}, l_{k} ; \lambda^{i}, \mathbf{l}^{i}\right)=Q^{k}\left(\lambda_{k}, \lambda^{i}\right)+L\left(l_{k} ; \tilde{l}_{k}\right) \\
& -\beta_{1} \Omega_{1}\left(\lambda_{k}, \lambda_{-k}^{i} ; l_{k}, 1_{-k}^{i}\right)-\beta_{2} \Omega_{2}\left(l_{k}, \mathbf{l}_{-k}^{i}\right)
\end{aligned}
$$

where $\lambda_{-k}^{i}$ and $l_{-k}^{i}$ are the vectors of length $(p-1)$ obtained by removing the $k$ th element from $\lambda$ and l. SAGE algorithms monotonically increase of both $\phi^{k}\left(\lambda_{k}, l_{k} ; \lambda^{i}, \mathbf{l}^{i}\right)$ and $\boldsymbol{\Phi}_{\text {joint }}(\lambda, 1)$. However the SAGE here does not guarantee global convergence since the penalty terms are nonconvex due to the discrete label parameters. To avoid getting stuck in local maxima, we use a deterministic annealing procedure for $\beta_{2}$, i.e. $\beta_{2}$ is increased exponentially as a function of iteration number until a specified value is reached.

\section{SIMULATIONS}

We have performed some preliminary simulations to compare the results from the joint estimation method and other image reconstruction methods in terms of both image visual quality and bias vs. variance relationship. A simple parallel collimated SPECT imaging system is simulated, with a 72-bin detector and 90 sampling angles over the 180-degree range. The image field is discretized into $128 \times 64$ pixels. The detector response is triangular with 4 pixel FWHM. We ignore the attenuation. All of each SPECT projection sets have about 1 million counts with $5 \%$ assumed known scatter; Poisson noise is simulated. We assume the MRI has been correctly segmented and registered. Fig.2(a) is the phantom for SPECT tracer distribution, Fig.2(b) is a segmented MRI with anatomical information perfectly matching the SPECT tracer distribution. Fig.2(c) is a segmented MRI with mismatched anatomical information, the hot elliptical region is missing.

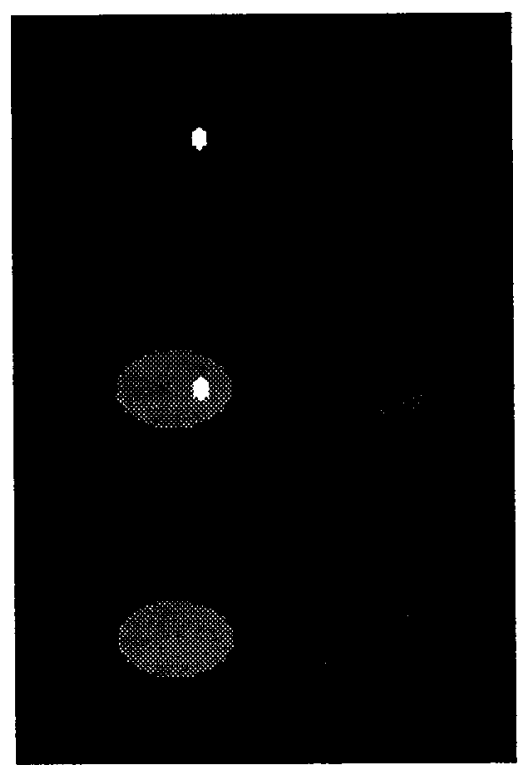

Figure 2: Top:(a) The phantom of SPECT tracer distribution. Middle:(b) Simulated segmented MRI region map with perfect side information. Bottom:(c) Simulated segmented MRI region map with missing side information.

Figure 3 shows images reconstructed from methods other than joint estimation. (a) is a filtered-backprojection with Generalized Hamming window, at $f_{c}=.80$ and $\alpha=.65$. Fig.3(b) through Fig.3(d) are from penalized SAGE with quadratic weighted Gibbs penalty. (b) is without any anatomical information, using uniformly weighted quadratic Gibbs penalty. In order to reduce noise, the image has to be globally regularized, so it looks blurry across all the region boundaries. In (c) the Gibbs weights were defined by the perfect MRI anatomical regions, (Fig.2(b)). Thus the regions are smooth, and boundaries are sharp. This shows the ultimate (also unrealistic) performance of applying MRI side information. In Fig.3(d), Fig.2(c) was used as side information to define the weights in the penalty term. Where the anatomical regions match the SPECT functional region, the reconstruction is as good as Fig.3(c), where mismatch exists, then the hot region in SPECT is blurred about the same amount as in Fig.3(b) where the penalty is uniformly weighted.

For joint estimation, penalized SAGE is applied to update each pixel-label pair $\left(\lambda_{k}, l_{k}\right)$ in four alternative raster scan orders. An idealized $L(\mathbf{l} ; \tilde{\mathbf{l}})$ table is used, see Table I. $\lambda$ is initialized with ramp filtered FBP, and $\mathbf{l}$ is initialized with Fig.2(b) or Fig.2(c) representing matched and mismatched side information. Fig.4(a) and (b) shows the SPECT reconstructed image and corresponding label estimation when the label is initialized with Fig.2(b). $\beta_{1}=2^{-8}$ and $\beta_{2}=2^{4.5}$ and they are fixed during the joint estimation process. This shows that when perfect MRI side information is available, the joint estimation also performs as well as Fig.3(c). For Fig.4(c) and (d), the 1 is initialized with Fig.2(c), a fixed $\beta_{1}=2^{-8}$ and an exponentially in- 


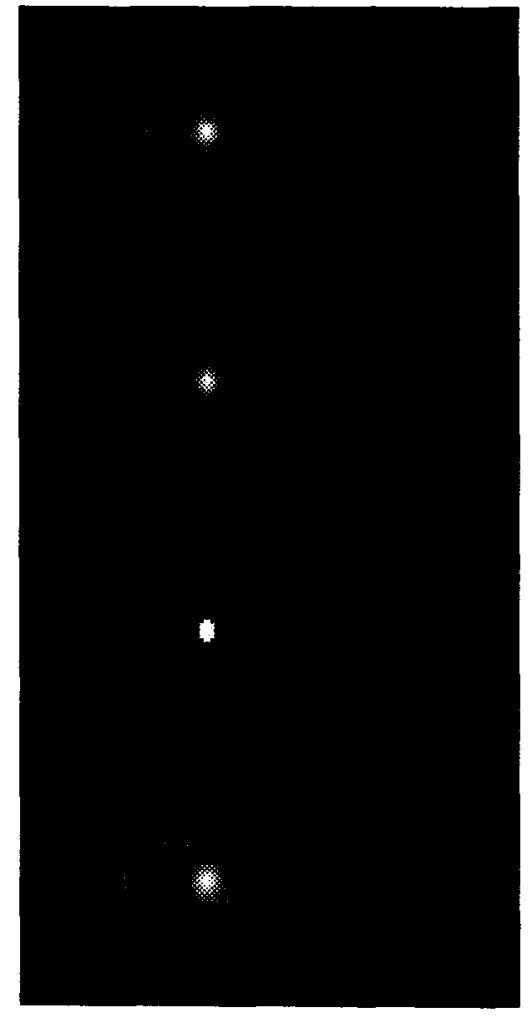

Figure 3: Top to bottom:a,b,c,d. (a):FBP reconstruction. (b):SAGE reconstruction with uniform 8 neighbor regularization. (c):SAGE with perfect MRI anatomical region information. (d):SAGE with mismatched MRI anatomical information.

creased $\beta_{2}=2^{4.5} \times 2^{\min (3, \text { iter })}$, where iter is the iteration index, and $\beta_{2}$ stays at the final value. Even though there was not any information associated with the hot region, the joint estimation recovers the labels for that region and helps to prevent the blurring observed in both Fig.3(c) and Fig.3(d). The newly estimated region labels are basically dependent on SPECT data, so they are not the same as the true region labels, due to the noise in SPECT data.

Bias vs. variance trade-offs are evaluated to avoid anecdotal performance comparison among different reconstruction methods. 50 SPECT noisy projection realizations are created. We evaluate the bias vs. standard deviation(std) for the hot region in terms of the concentration in that region. For the cases where the perfect MRI side information is available, we use the ideal ROI label to define the region. For joint estimation with mismatched MRI side information, we use estimated labels to define that region. For uniform regularization and fixed application of MRI cases, we use $75 \%$ of the peak value in a bigger region containing the ROI as a threshold to define the estimated ROI. The concentration is then the averaged counts per pixel in that defined region. Fig. 5 shows the bias vs. STD comparison. When the ideal and perfectly matched MR anatomical information is used for the penalty, it gives ultimate performance, i.e. for given standard deviation, it

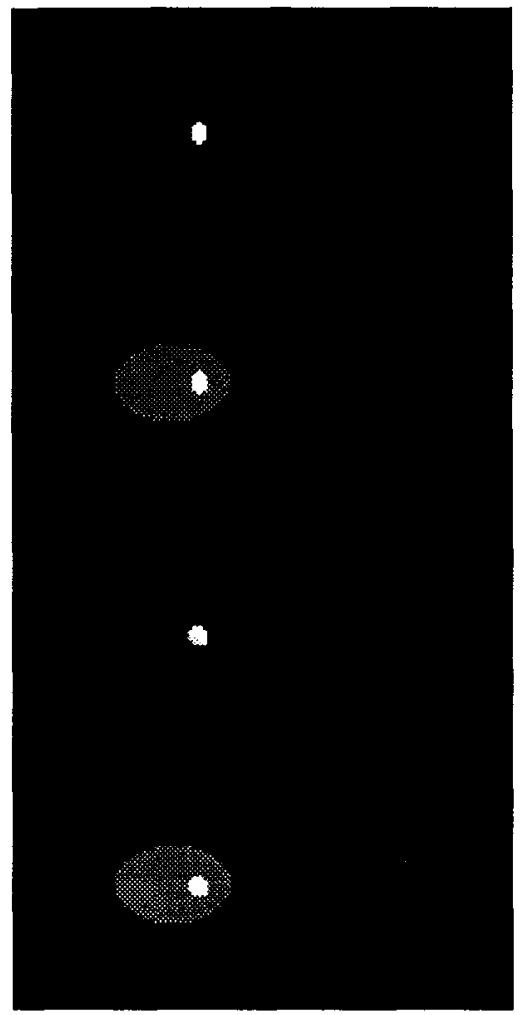

Figure 4: Top to bottom:a,b,c,d. (a:image,b:label) joint estimation initialized with perfect MRI information. (c:image,d:label) joint estimation initialized with mismatched MRI.

has the smallest bias(case 1). On the contrary, when there is no MRI side information, we can only apply uniform regularization to control the trade-off between image resolution and noise. For given standard deviation, this gives the highest bias(case 2). If MRI information has some mismatch, such as the missing ROI in case 3, fixed application of this MRI information will give the artifact blurring in the reconstructed SPECT image, so the performance is close to case 2. When the joint estimation is initialized with perfect MRI anatomical information, the performance is very close to the ultimate performance of the PML with perfect side information(case 4). This shows the potential of the joint estimation approach. Even with mismatched side information, joint estimation is able to partially recover the region and prevent blurring across the region boundaries, so that the estimation performance (case 5) of concentration in that region is better than case 1 and 2 .

However, we have observed that the convergence is sensitive to the initial $\boldsymbol{\lambda}$ and $\left(\beta_{1}, \beta_{2}\right)$ pair, i.e. for some initial $\lambda$, only a small range of $\beta_{1}, \beta_{2}$ will give convergence to a local maximum close to the global maximum, otherwise the joint estimation of both $\hat{\boldsymbol{\lambda}}$ and $\hat{\mathbf{I}}$ will converge to a local maximum far from the global maximum, as shown in Fig.6. 


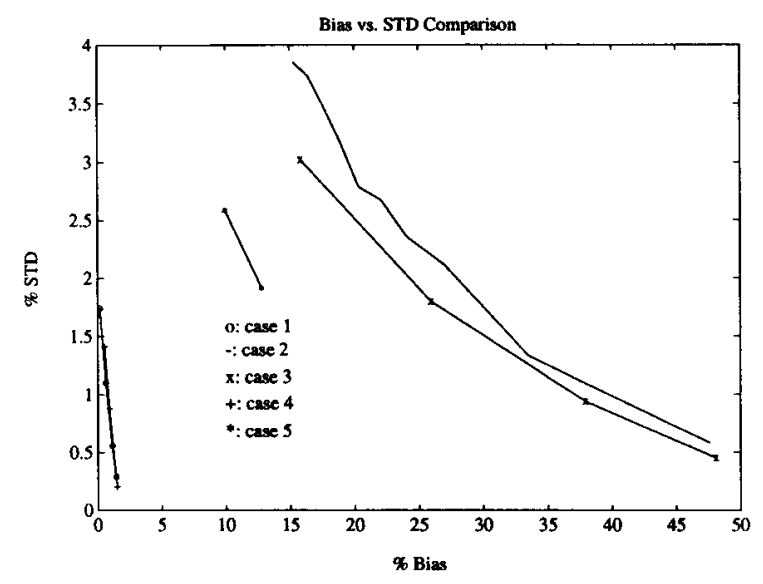

Figure 5: Bias vs. STD in terms of the concentration the given $\mathrm{ROI}$.

\section{DISCUSSION}

We have shown that the joint estimation approach preserves the benefit of applying the correlated anatomical side information, so the bias and variance are reduced. It can also avoid being dominated by the mismatched region information. So the joint estimation approach has the potential of robustly incorporating MRI side information.

We have also noticed some problems that will be addressed in the future study. First, the objective function is non-concave, because discrete label parameters are estimated together with continuous SPECT intensity parameters. This destroys the global convergence property of the SAGE algorithm [6]. We have shown that in Fig.6. Since the non-concavity is inherent, we have to find a way of choosing the initial image and best $\left(\beta_{1}, \beta_{2}\right)$ pair. Further algorithm development is needed. Second, the MRI measurement distribution table used in simulation is an idealized and simplified one. We assumed a simple pixelby-pixel segmentation scheme, but if the MRI is segmented using a more sophisticated approach such as ICM algorithm $[8]$, then $L(\mathbf{l} ; \tilde{\mathbf{1}})$ will be very difficult to determine. At present that some human observation of both FBP and MRI is needed to determining whether there possibly exists a region in SPECT which does not appear in MRI. To obtain this information more automatically and robustly is also a future study.

\section{REFERENCES}

[1] X. Yan, R. Leahy, "MAP Image Reconstruction Using Intensity and Line Processes for Emission Tomography Data" SPIE: Image Processing Algorithms and Techniques $I I$, vol. 1452, pp. 158-168, 1991.

[2] S. Geman, D. Geman, "Stochastic Relaxation, Gibbs Distribution and the Bayesian Restoration of Images", IEEE Trans. on Pattern Analysis and Machine Intelligence, Vol PAMI-6 No.6 Nov. 1984.

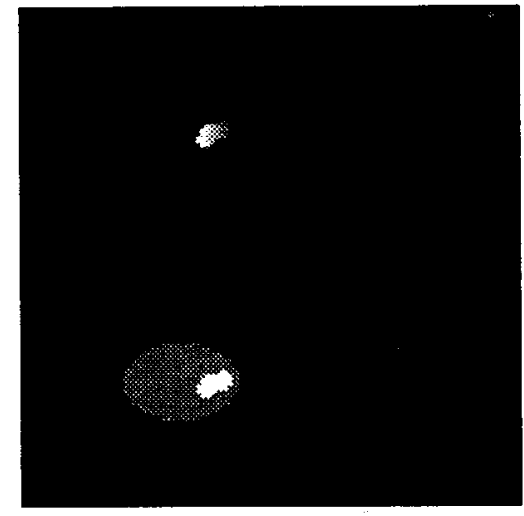

Figure 6: Both $\boldsymbol{\lambda}$ (top) and $\mathbf{l}$ (bottom) converge to a local maximum when joint estimation is initialized with mismatched MRI information.

[3] J. Fessler, N. Clinthorne, L. Rogers, "Regularized Emission Image Reconstruction Using Imperfect Side Information", IEEE Trans. on Nuclear Science, Vol. 39, pp. 1464-1471, No.5, Oct. 1992.

[4] V. Johnson, J. Bowsher, R. Jaszczak, and T. Turkington, "Analysis and Reconstruction of Medical Images Using Prior Information", Discussion Papers Institute of Statistics and Decision Sciences, Duke University, 1993.

[5] X. Ouyang, W.H. Wong, V. Johnson, X. Hu, and C.T. Chen, "Incorporation of Correlated Structural Images in PET Image Reconstruction" Pre-print

[6] J.A. Fessler, A.O. Hero, "Space-Alternating Generalized Expectation-Maximization Algorithm", IEEE Trans. on Signal Processing Vol. 42, No. 10, Oct. 1994

[7] K. Lange and R. Carson, "EM Reconstruction Algorithms for Emission and Transmission Tomography", Journal of Computer Assisted Tomography, 8(2): 306316, April 1984.

[8] J. Besag, "On the statistical analysis of dirty pictures", Journal of the Royal Statistical Society Series B, 48(3), 1986.

\begin{tabular}{||l|l|l|l|l||}
\hline$p(0 \mid 0)=1$ & $p(0 \mid 1)=0$ & $p(0 \mid 2)=0$ & $p(0 \mid 3)=0$ & $p(0 \mid 4)=0$ \\
\hline$p(1 \mid 0)=0$ & $p(1 \mid 1)=1$ & $p(1 \mid 2)=0$ & $p(1 \mid 3)=0$ & $p(1 \mid 4)=0$ \\
\hline$p(2 \mid 0)=0$ & $p(2 \mid 1)=0$ & $p(2 \mid 2)=1$ & $p(2 \mid 3)=0$ & $p(2 \mid 4)=0$ \\
\hline$p(3 \mid 0)=0$ & $p(3 \mid 1)=0$ & $p(3 \mid 2)=0$ & $p(3 \mid 3)=1$ & $p(3 \mid 4)=1$ \\
\hline
\end{tabular}

Table I: Ideal probability distribution of $P\left(\tilde{l}_{k} \mid l_{k}\right)$. 